\title{
Inferring Image Transformation and Structure from Motion-Blurred Images
}

\author{
Paramanand Chandramouli \\ paramanand@gmail.com
}

\author{
A. N. Rajagopalan \\ raju@ee.iitm.ac.in
}

\author{
Image Processing and Computer Vision Lab \\ Department of Electrical Engineering \\ Indian Institute of Technology Madras \\ Chennai, India
}

\begin{abstract}
This paper deals with the problem of estimating structure of 3D scenes and image transformations from observations that are blurred due to unconstrained camera motion. Initially, we consider a fronto-parallel planar scene and relate the reference image of the scene to its motion-blurred observation by finding the reference image transformations. The blur kernel at every image point can be determined from these transformations. For $3 \mathrm{D}$ scenes, the extent of blurring in the image is related to the camera motion as well as the scene structure. We propose a technique to estimate the scene depth with the knowledge of the estimated image transformations. The proposed method is validated by testing on real and synthetic experiments.
\end{abstract}

\section{Introduction}

While capturing images of a scene, the relative motion between the camera and the scene during exposure leads to motion-blur in images. As the camera moves, the sensors are exposed to different scene points and the resulting image is the average of the light intensities observed by the sensors. The extent of camera motion determines the extent of smearing in the blurred image. The motion-blurred image is regarded as the degraded version of the reference image of the scene which is captured by a stationary camera and is usually modeled as the convolution with a space-invariant blur kernel [曰, 四].

The convolution model is applicable only when the camera motion is restricted to inplane translations. Figs. 1 (a) and (b) show two observations of a planar scene captured by a camera. The image of Fig. 1 (a) was captured when the camera was still, while Fig. 1 (b) was captured when the camera was moved along the optical axis during the exposure. In Fig. 1 (b), we observe that the extent of blurring differs across the image. In regions farther from the image center, the smearing is more than in regions that are close to the image center. Such degradations cannot be considered as the convolution of the reference image with a single blur kernel $[\square, \mathbb{}, \mathbb{}$ ] $]$. In this paper, we model the motion-blurred image as the weighted average of geometrically transformed versions of the reference image. The model implicitly accounts for the space-variant nature of blurring that occurs due to unrestricted camera motion.

In many applications, motion-blur can occur while capturing scenes with depth variations from a moving camera. During the camera motion, the apparent movement of the scene 


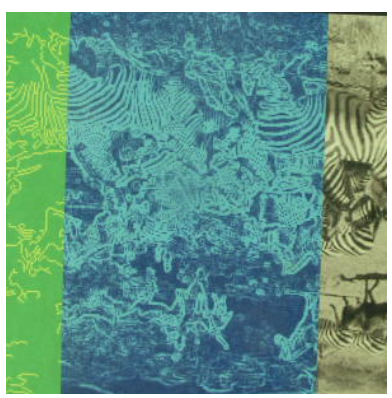

(a)

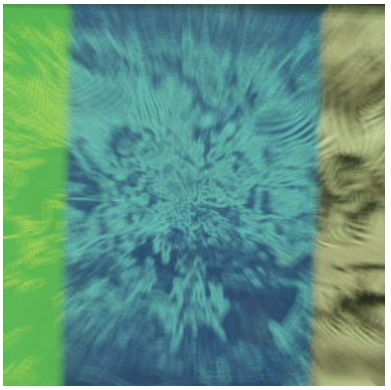

(b)

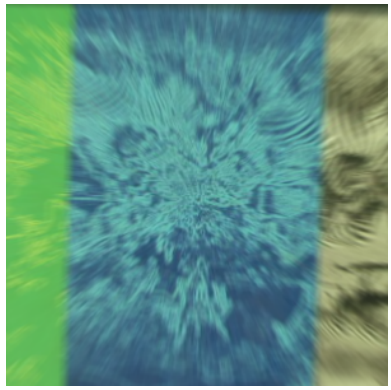

(c)

Figure 1: (a) Reference image. (b) Blurred observation. (c) Transformed reference image.

points in the image is related to the shape of the scene [ $\mathrm{\nabla}]$. Consequently the extent of blurring at a point is governed by both the camera motion and the scene structure. We develop a technique to estimate the image transformations and recover the shape of the scene from the motion-blurred observation. The objective of our work is similar to that of structure from motion algorithms which estimate the camera motion as well as the scene structure from a sequence of images [Q].

\subsection{Related work}

Several works exist in the literature that consider space-variant blur in images due to camera or object motion. Techniques that address blurring due to rotation for performing image restoration include $[\boldsymbol{\theta}, \mathbb{\square}, \square]$. Motion-blurred images have been used to estimate the rotational velocity from a single observation in $[\square, \square]$. In [四, the 3D direction of camera translation is recovered from a motion-blurred image by estimating the direction and extent of smearing across the image.

Mei and Read [四] have proposed a tracking algorithm in the presence of complicated motion-blur. They relate the current observation and the reference image through a homography transformation and consider that the current observation is blurred due to camera motion along an arbitrary direction. The transformation is estimated for each frame and the reference unblurred image is aligned with the blurred observation. They make an assumption that the camera velocity is uniform during exposure. In this paper, we relax the uniform velocity assumption. In [छ], Whyte et al. propose an image restoration technique for motion-blur arising due to non-uniform camera rotations. They represent the blur kernel on a three dimensional grid corresponding to the three directions of camera rotations about its center. For the case of blind image restoration, they use an existing framework of blind kernel estimation [छ]. When a noisy version of the original image is available, they use a least-squares energy minimization approach for finding the blur kernel. The motion-blur model we use in this paper is similar to their approach. We consider camera translations in all three directions and in-plane rotations.

Motion-blurred images have been used for depth estimation in [ $\boldsymbol{\theta}]$ and [ $\square]$. These methods assume that the scene is of constant depth or can be approximated by a set of planar patches. Depth estimation and restoration for space-variant blurred images is performed us-

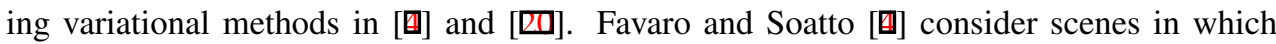
different objects move along different directions. They estimate the motion field, depth-map 
and the restored image from the motion-blurred observations which are captured with different exposure times. Sorel and Flusser have proposed a technique to estimate the shape and the restored image using two observations which are blurred in different ways [ $\mathbb{}$ ] $]$. They consider the PSF to be of arbitrary shape (due to non-uniform velocity of the camera). In their method, the PSF is initially determined from the blurred observations by choosing regions of constant depth. Using this PSF, the image and the depth-map are simultaneously estimated. Our work in [ㅁ] uses two observations of the scene and determines the depth at a point by estimating extent of relative blur between the observations. The relation between the depth at a point and the blurred observation is shown to be nonlinear. Depth estimation is formulated as a recursive state estimation problem and is solved using an unscented Kalman filter (UKF) [ $[$ ]. In all these depth estimation techniques, the relative motion between the camera and scene was restricted to fronto-parallel translations wherein the extent of blurring at a point is directly related to the scene depth. However, realistic camera shakes include even in-plane rotations [ $\square]$. When there are in-plane rotations, the extent of blurring at a point also depends on its location with respect to the center of rotation.

In this paper, we develop a method to estimate the transformations induced by the camera motion and the structure of the scene from a reference image and a blurred observation. In the initial part of our work, we consider scenes having constant depth and model the spacevariant blurring due to general camera motion as the averaging of the warped instances of the reference image. The model does not assume uniform camera velocity and can account for arbitrary handshakes. We develop an algorithm to estimate the transformations undergone by the reference image during exposure. This algorithm can be used to align a reference template with its space-variantly blurred observation. The transformations considered are restricted to translations, rotations and scale changes. However, our framework can be extended to homographies. We next consider images of scenes with depth variations. We select regions of constant depth in the reference and the blurred images (as done in $[\mathrm{W}]$ ) to estimate the transformations induced by the camera motion. Based on this information, we relate the depth at a scene point to the blurred image intensity through the point spread function. Depth estimation is posed as a state estimation problem and is solved in a UKF-based approach.

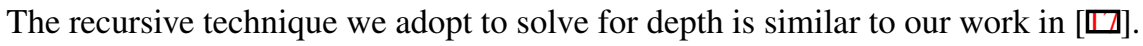

\section{Motion-blur model}

In this section, we discuss the modeling of motion-blurred image in terms of the reference unblurred image of the scene. We assume that all points in the scene have the same depth. A motion-blurred image $g$ can be related to the reference image $f$ through the space-variant point spread function (PSF) $h$ as

$$
g(x, y)=f *_{\nu} h(x, y)=\int_{-\infty}^{+\infty} \int_{-\infty}^{+\infty} f(x-s, y-t) h(x-s, y-t ; s, t) d s d t
$$

where $*_{v}$ denotes space-variant blurring operation. For the case of in-plane camera translations, the PSF remains constant at all the image points. However, when the camera motion is not restricted to in-plane translations, the PSF can vary at every image point. We model the motion-blurred image in terms of the reference image using a function which we call as transformation spread function (TSF). TSF denotes a weighted collection of the transformations the image points undergo during the exposure. The PSF at every image point can be obtained from the knowledge of TSF. 
The PSF at a point $(x, y)$ denoted by $h(x, y, ;)$ can be shown [四] to be

$$
h(x, y, s, t)=\frac{1}{T_{e}} \int_{0}^{T_{e}} \delta(s-\bar{x}(x, y, \tau), t-\bar{y}(x, y, \tau)) d \tau
$$

where $T_{e}$ is the total exposure duration, $\delta$ indicates the two dimensional Dirac Delta function, and $\bar{x}(x, y, \tau)$ and $\bar{y}(x, y, \tau)$ denote the components of the displacement of the point $(x, y)$ during the exposure. The PSF represents the displacements of the image point $(x, y)$ during the exposure time and is weighted according to the fraction of the exposure time the point stays at the displaced position. For instance, during the exposure, if the image undergoes translations $\left(s_{a}, t_{a}\right)$ for some duration and $\left(s_{b}, t_{b}\right)$ for the remaining duration, the PSF at all points is given by $h(x, y, s, t)=w_{a} \delta\left(s-s_{a}, t-t_{a}\right)+w_{b} \delta\left(s-s_{b}, t-t_{b}\right)$, where $w_{a}$ and $w_{b}$ correspond to the exposure durations at the translations $\left(s_{a}, t_{a}\right)$ and $\left(s_{b}, t_{b}\right)$, respectively. When the camera is free to rotate or translate in any direction, different image points are displaced by different extents resulting in space-variant PSFs.

Let $\mathbf{T}$ denote the set of possible geometric transformations the image points can undergo during the exposure. We define the transformation spread function $h_{T}: \mathbf{T} \rightarrow \mathfrak{R}_{+}$as a mapping from the set of transformations to the set of nonnegative real numbers. $h_{T}\left(T_{\lambda}\right)$ denotes the fraction of the exposure duration for the image transformation $T_{\lambda}$. The blurred image is modeled as the weighted sum of the transformed reference images.

$$
g=\sum_{T_{\lambda} \in \mathbf{T}} h_{T}\left(T_{\lambda}\right) f_{T_{\lambda}}
$$

where $f_{T_{\lambda}}$ denotes the reference image warped by the transformation $T_{\lambda}$. According to this model, during the exposure time, the reference image undergoes different geometric transformations $\left(T_{\lambda} s\right)$ and these transformed images are weighted according to their exposure durations. The resultant blurred image is the weighted average of the transformed images. Analogous to the PSF, the TSF satisfies the relation $\sum_{T_{\lambda} \in \mathbf{T}} h_{T}\left(T_{\lambda}\right)=1$ when the exposure times are equal for the reference and blurred images.

In this work, we assume that the camera motion is restricted to translations along all three directions and in-plane rotations (about the optical axis). For fronto-parallel planar scenes, such camera motions result in translations, rotations and scaling of the image points. The set $\mathbf{T}$ is regarded as a subset of a four dimensional space parameterized by translation, rotation and scale factors. Let the translation, rotation and scale axes of the set of $\mathbf{T}$ be denoted by $t_{x}, t_{y}, t_{\theta}$ and $t_{s}$, respectively. The TSF depicts the camera motion during the exposure. For instance, if the camera motion is restricted to rotations about the optical axis during the exposure, the PSF will vary at every image point depending on its distance from the center of rotation and camera velocity. Whereas, the TSF representation contains non-zero components only along the $t_{\theta}$ axis, which corresponds to the camera motion. Similarly, the components along $t_{x}, t_{y}$, and $t_{s}$ axes indicate the camera translations.

Let $h(i, j, ;)$ denote the discrete PSF at the image point $(i, j)$. Let $\left(i_{\lambda}, j_{\lambda}\right)$ denote the co-ordinates of the point when a transformation $T_{\lambda}$ is applied on $(i, j)$. We can obtain the PSF at each pixel $(i, j)$ from TSF (which is common for all the image points) as

$$
h(i, j ; m, n)=\sum_{T_{\lambda} \in \mathbf{T}} h_{T}\left(T_{\lambda}\right) \delta_{d}\left(m-\left(i-i_{\lambda}\right), n-\left(j-j_{\lambda}\right)\right)
$$

where $\delta_{d}$ denotes the $2 \mathrm{D}$ Kronecker delta function. When the points $\left(i_{\lambda}, j_{\lambda}\right)$ take non-integer values, bilinear interpolation is used. From Eqn. (4), It is easy to see that if $\mathbf{T}$ is restricted to translations alone, then the TSF and PSF are identical. 
We would like to mention that, the transformations we have considered form a subspace of the 2D affine transforms around a neighborhood of the identity transform in the Lie algebraic representation [ $[\mathbb{}]$ ]. In order to allow more degrees of freedom for the camera motion, the transformations have to be represented in a higher dimensional space, as done in [एర]].

\section{Estimating TSF}

We now discuss the estimation of TSF when the reference image $f$ and the blurred observation $g$ are given. The transformation space is sampled to get a discrete set of transformations. Let $N_{x}, N_{y}, N_{\theta}$ and $N_{z}$ be the number of quantization levels in the axes $t_{x}, t_{y}, t_{\theta}$ and $t_{s}$, respectively. The TSF $h_{T}$ can be considered as vector in $\mathfrak{R}^{N_{T}}$ (denoted as $\mathbf{h}_{T}$ ) with each of its components representing the weight, and $N_{T}=N_{x} N_{y} N_{\theta} N_{z}$. In practice, $\mathbf{h}_{T}$ will be a sparse vector because the reference image would undergo very few transformations out of $\mathbf{T}$ during the image capture. $\mathbf{h}_{T}$ is estimated by minimizing the error between the blurred observation $g$ and the transformed reference image $f$ according to Eqn. (3)

Let $f$ and $g$ be of size $N_{p} \times N_{p}$ pixels and the PSF at each point be of size $N \times N$. Space variant blurring can also be expressed as

$$
\bar{g}=F \bar{h}
$$

where $\bar{g}$ is a $N_{p}^{2} \times 1$ vector, $F$ is a sparse matrix of size $N_{p}^{2} \times N_{p}^{2} N^{2}$, and $\bar{h}$ is of size $N_{p}^{2} N^{2} \times 1$ formed by stacking the blur kernel $h(i, j, ;)$ at every pixel. We estimate the TSF by minimizing the following cost function using gradient descent technique.

$$
\begin{gathered}
L\left(\mathbf{h}_{T}\right)=\left\|g-\sum_{T_{\lambda} \in \mathbf{T}} h_{T}\left(T_{\lambda}\right) f_{T_{\lambda}}\right\|^{2}=\|\bar{g}-F \bar{h}\|^{2} \\
\frac{\partial L\left(\mathbf{h}_{T}\right)}{\partial \mathbf{h}_{T}}=\frac{\partial \bar{h}}{\partial \mathbf{h}_{T}} \frac{\partial L}{\partial \bar{h}}=2 \frac{\partial \bar{h}}{\partial \mathbf{h}_{T}} F^{T}(F \bar{h}-\bar{g})
\end{gathered}
$$

The gradient with respect to the PSF can be evaluated from Eqn. (4). We avoid explicit matrix multiplications by using Eqn. (3) for blurring operation. In our experiments, we restrict the value of $N_{T}$ to be much lesser than the total number of image pixels in order to have enough number of observation for getting an accurate least-squares estimate. The algorithm converges even when all the $N_{T}$ transformations are equally weighted in the initial estimate of $\mathbf{h}_{T}$.

\section{Structure estimation from motion-blur}

In scenes with depth variations, the extent of blurring at a point depends both on the scene structure and the camera motion. We see that blur can serve as a depth cue when there is a translational component in the camera motion. In this section, we discuss the blurring model for 3D scenes using TSF and the technique to estimate depth from the reference image and the blurred observation. For the sake of simplicity, we restrict to in-plane camera translations and rotations in this section. 


\subsection{Effect of depth on blur}

In the reference image $f$ consider a patch of image pixels $f_{o}$ corresponding to a region of constant depth $d_{o}$. Let $g_{o}$ be the corresponding patch in the blurred image $g$. Let $h_{T_{o}}$ be the transformation spread function which yields $g_{o}$ when applied on $f_{o}$. Consider an image point $\mathbf{p}_{o}=\left[i_{o} j_{o}\right]^{T}$ in the reference patch. When the camera undergoes an in-plane rotation and translation during exposure, let the resulting transformation of the points in the reference patch be $T_{\lambda}$. We can write $T_{\lambda}$ as a rotation operation $R_{\lambda}$ () followed by a translation $\mathbf{t}_{\lambda}=\left[\begin{array}{ll}\tau_{\lambda_{x}} & \tau_{\lambda_{y}}\end{array}\right]^{T}$. The point $\mathbf{p}_{0}$ shifts to $\mathbf{p}_{o}^{\prime}=R_{\lambda}\left(\mathbf{p}_{o}\right)+\frac{\mu \mathbf{t}_{c_{\lambda}}}{d_{o}}$ where $\mathbf{t}_{c_{\lambda}}$ denotes the camera translation, and $\mu$ denotes the 'focal length' of the imaging system. The translation of the image point $\mathbf{t}_{\lambda}=\frac{\mu \mathbf{t}_{c}}{d_{o}}$. We note that the rotation operation would have the same effect even if the value of the reference depth were to be different. In contrast, the extent of translation a point undergoes is dependent on its depth. For the same camera motion, the point $\mathbf{p}=[i j]^{T}$ would have shifted to $\mathbf{p}^{\prime}=R_{\lambda}(\mathbf{p})+\frac{\mu \mathbf{t}_{c_{\lambda}}}{d(i, j)}=R_{\lambda}(\mathbf{p})+\frac{\mathbf{t}_{\lambda}}{d(i, j) / d_{o}}$, where $d(i, j)$ denotes the depth of p. During the exposure, the camera undergoes different translations and rotations resulting in the blurred image. The variation of blurring with respect to depth happens due to translational component of camera motion.

Suppose we know the reference TSF $h_{T_{o}}$ (determined at depth $d_{o}$ ), the PSF at every point can be related to its depth value. If a point in the reference patch (having depth $d_{o}$ ) undergoes a transformation $T_{\lambda}$ composed of rotation $R_{\lambda}$ and translation $\mathbf{t}_{\lambda}$, then a point $\mathbf{p}_{(i, j)}$ having depth $d(i, j)$ would undergo a rotation $R_{\lambda}$ and translation $\frac{\mathbf{t}_{\lambda}}{k(i, j)}$, where $k(i, j)=\frac{d(i, j)}{d_{o}}$ denotes the relative depth at $(i, j)$. Using this fact, we can write the expression for the PSF at a point $(i, j)$ as

$$
h(i, j ; m, n)=\sum_{T_{\lambda} \in \mathbf{T}} h_{T_{o}}\left(T_{\lambda}\right) \delta_{d}\left(m-\left(i-i_{\lambda}\right), n-\left(j-j_{\lambda}\right)\right)
$$

where $\left[i_{\lambda} j_{\lambda}\right]^{T}=R_{\lambda}\left(\mathbf{p}_{(i, j)}\right)+\frac{\mathbf{t}_{\lambda}}{k(i, j)}$. The blurred image $g$ is given by

$$
g(i, j)=\sum_{m, n} f(i-m, j-n) h(i-m, j-n ; m, n)
$$

From Eqns. (8) and (9), we get the relationship between the relative depth at a point and the intensity of the blurred image through the PSF.

\subsection{UKF-based depth estimation}

For the case of in-plane camera translations, the shape of the PSF remains constant at all the image points except for a scale factor which is related to the scene depth. Points that are near the camera are more blurred than those that are farther. Based on the extent of blurring at a point, the depth values are estimated $[\square, \square]$. However, in the presence of camera rotations, points that are farther from the center of rotation are more blurred than those that are close to the center, irrespective of depth. Hence, the extent of blurring does not directly reflect the depth at a point. We use the knowledge of TSF at the reference region to estimate the relative

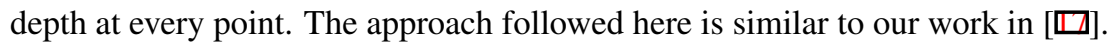

Initially, we determine the TSF $h_{T_{o}}$ from a patch $f_{o}$ of the reference image $f$ and the corresponding patch $g_{o}$ of $g$ using the proposed TSF estimation technique. We consider it as the reference TSF and assume that its depth is $d_{o}$. We estimate the relative depth 
$k(i, j)=d(i, j) / d_{o}$ at every $(i, j)$ by relating it to the blurred image intensity. In order to facilitate recursive estimation of $k(i, j)$, we locally approximate the blurring model of Eqn.

(9) as

$$
g(i, j)=\sum_{m, n} f(i-m, j-n) h(i, j ; m, n)+e(i, j)
$$

where $e()$ is assumed to be additive Gaussian noise with zero mean and variance $\sigma_{e}^{2}$. According to this model, the blurred image intensity $g(i, j)$ is dependent on the reference image $f$, the reference TSF $h_{T_{o}}$, and the scale factor $k(i, j)$. We estimate $k(i, j)$ with the knowledge of $f, g$ and $h_{T_{o}}$ by posing it as a recursive state estimation problem. To handle the nonlinear relationship between the state $k(i, j)$ and the observation $g(i, j)$, we use an unscented Kalman filtering approach. Due to space constraints, we give a summary of the recursive filtering technique that we followed. More details can be found in [प]

Kalman filtering requires propagation of mean and covariance of the state estimate through the system and observation models. We use a discontinuity adaptive Markov random field (DAMRF) prior [ $\square$ ] for the system model. The DAMRF prior helps in incorporating smoothness, and in adapting the estimation process at the edges. The measurement model used is

$$
\mathbf{g}(i, j)=\mathbf{H}_{i, j}(k(i, j))+e(i, j)
$$

where $\mathbf{g}(i, j)$ denotes blurred image pixels corresponding to all three channels and the nonlinear operator $\mathbf{H}_{i, j}$ is in terms of $f$ and $h_{T_{o}}$. At each pixel $(i, j)$, the state mean and covariance are predicted through the system model. From these, the observation moments are obtained through unscented transformations [四]. The Bayesian estimate of the state mean is updated based on the observation through the UKF. The updated mean is regarded as the relative depth $k(i, j)$.

\section{Experimental results}

To evaluate the performance of the proposed technique, we performed real and synthetic experiments. We initially performed a synthetic experiment to test the proposed TSF estimation technique. The reference image shown in Fig. 2 (a) was blurred by simulating the effect of axial camera motion. It was scaled by different scale factors and a weighted average of these scaled image was obtained. The scale factors and their weights are indicated in the transformation spread function in Fig. 2 (d). Along the $t_{s}$ axis of the TSF, the value of scale factors ranged from 0.8 to 1.2 in steps of 0.01 . Fig. 2 (b) shows the resultant motion-blurred image. We used the proposed TSF estimation technique to estimate the transformation between the reference and the blurred images. The estimated TSF shown in Fig. 2 (e) is very close to the actual TSF of Fig. 2 (d). When the reference image was transformed with this estimated TSF, we obtain the image shown in Fig. 2 (c). We see that the blurred image (Fig. 2 (b)) and the transformed image (Fig. 2 (c)) are very similar. The rms error between the transformed image and the blurred image was 0.21 which is quite small. In this experiment, the TSF was defined only on $t_{s}$ axis to avoid unnecessary computations.

We next tested the proposed TSF estimation technique on images captured by a camera. We considered the fronto-parallel planar scene shown in Fig. 1 (a) as the reference image. The blurred observation in Fig. 1 (b) was captured when the camera was moving along the optical axis during exposure. After estimating the TSF, the reference image was transformed to get the image shown in 1 (c). The transformed reference image and the blurred observation are close to each other. We also tested the algorithm when the reference image was 


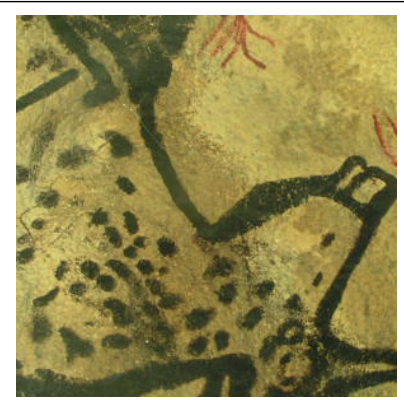

(a)

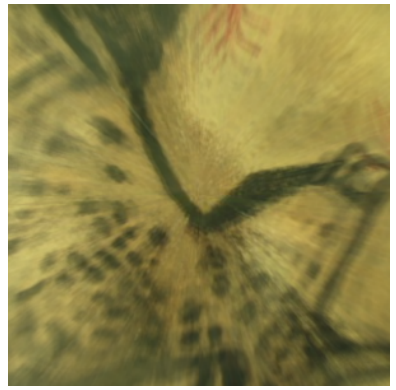

(b)

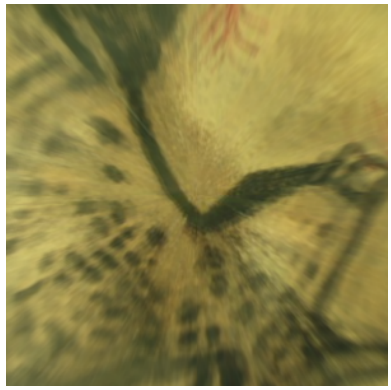

(c)

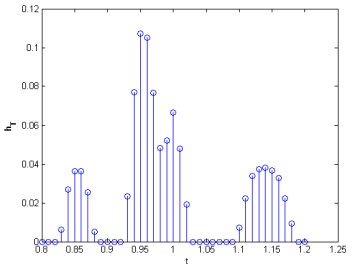

(d)

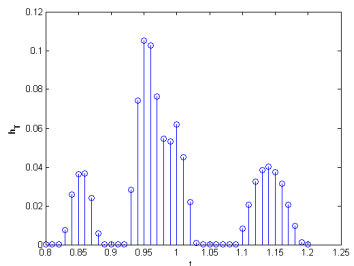

(e)

Figure 2: (a) Reference image. (b) Blurred observation. (c) Transformed reference image. (d) True TSF. (e) Estimated TSF.

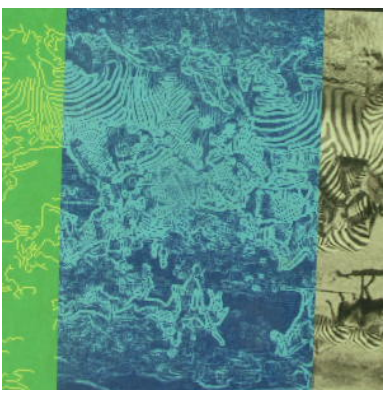

(a)

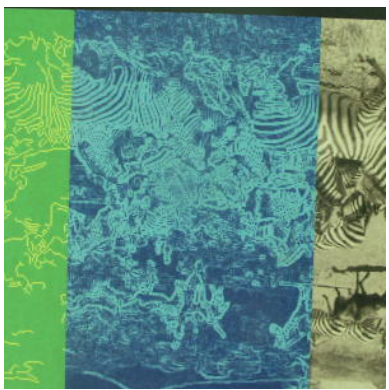

(b)

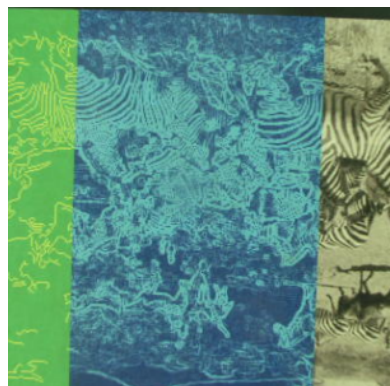

(c)

Figure 3: (a)Reference image. (b) Observation.(c) Warped reference image.

transformed by a single scale factor. We shifted the camera from its original position along the optical axis to get a scaled image which was regarded as the 'blurred observation'. The reference image and the scaled observation are shown in Figs. 3 (a) and (b) respectively. In the estimated TSF, a peak was observed at the scale factor 0.94 . The reference image was warped using the estimated TSF to get the observation shown in Fig. 3 (c). Comparing Figs. 3 (b) and (c), we see that the estimated transformation is correct.

In our next experiment, we tested the proposed TSF estimation and depth estimation techniques on synthetic data. The calf leather image ([G]) shown in Fig. 4 (a) was used as the reference image. The relative depth values for the scene were assumed to be varying from 1 to 2.5 as shown in Fig. 4 (e). We synthetically simulated camera in-plane translations and rotations by assuming a TSF ranging between -7 and 7 along both the translation axes, $-3^{\circ}$ and $3^{\circ}$ along the angle axis, and with scale factor as 1 at locations with relative depth value 1 . The reference image was blurred by generating the PSF at every point according to the TSF and the relative depth values to get the blurred image (Fig. 4 (b)). Initially, we 


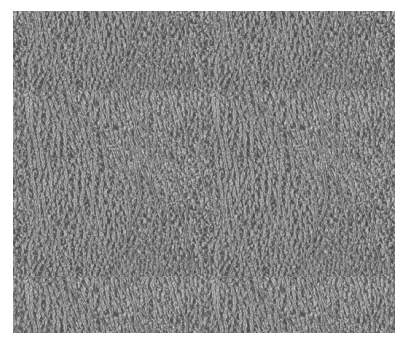

(a)

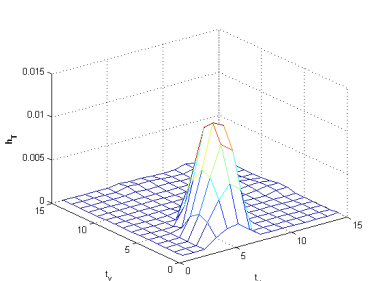

(d)

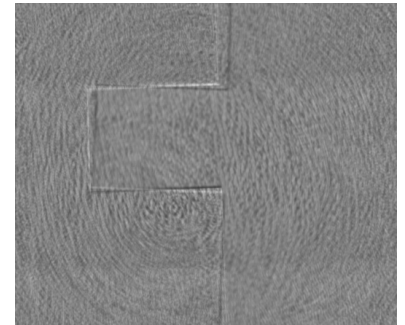

(b)

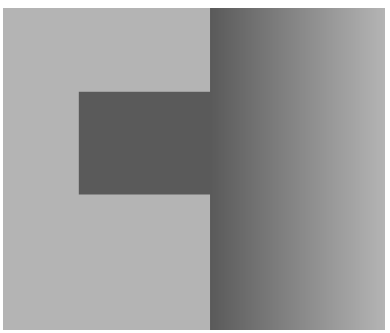

(e)

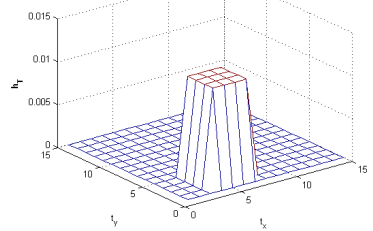

(c)

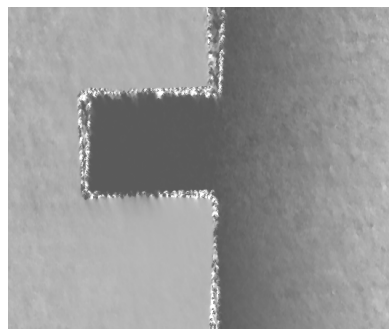

(f)

Figure 4: (a) Reference image. (b) Blurred observation. (c) and (d) True and estimated TSF at $t_{\theta}=-1^{\circ}$ and $t_{s}=1$, respectively. (e) True depth map. (f) Estimated depth map.

estimated the TSF using image patches from the reference and blurred images. In the initial estimate of TSF, all the transformations were weighted equally. Fig. 4 (d) shows a part of the estimated TSF for $t_{\theta}=-1^{\circ}$ and $t_{s}=1$. The corresponding part of the true TSF is shown in Fig. 4 (d). The estimated TSF was close to the true TSF since the rms error between the transformed reference image patch and blurred patch was 2.1. We applied the proposed UKF-based depth estimation technique using the estimated value of TSF to get the depthmap shown in Fig. 4 (f). The estimated depth-map and the true depth map are close to each other except at the sudden depth discontinuities. The rms errors calculated over patches in the ramp region and the plane region of the depth-map were 0.057 and 0.0085 , respectively. The overall rms error was 0.17 which by itself is quite low.

We next applied the proposed method on real images. The reference image of the scene consisting of three objects at different depths is shown in Fig. 5 (a). The blurred observation shown in Fig. 5 (b) was captured by both rotating and translating the camera during exposure. We selected patches of $200 \times 170$ pixels in the middle region of the green object from the reference and blurred images to estimate the reference TSF. Since the rotation extent was small, significant components in the TSF were observed at $1^{\circ}, 2^{\circ}$ and $3^{\circ}$. The estimated TSF for $t_{\theta}=3^{\circ}$ and $t_{s}=1$ is shown in Fig. 5 (c). We then performed the UKF-based depth estimation to estimate the relative depth values. The median-filtered depth map is shown in Fig. 5 (d). In Fig. 5 (d), we observe that the objects that are near the camera are correctly assigned a lesser relative depth value than those that are farther. In the blurred observation (Fig. 5 (b)), we see that the extent of blurring is more at the top left portion of the green object and less in the bottom right region due to the difference in the distance from the center of rotation. Despite these effects, the estimated depth values of all the points of the green object are nearly equal, as to be expected. 


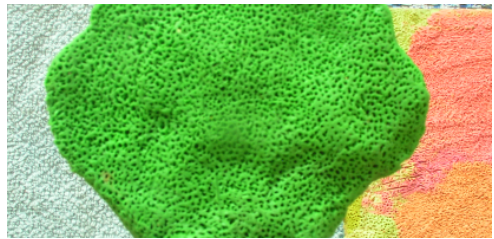

(a)

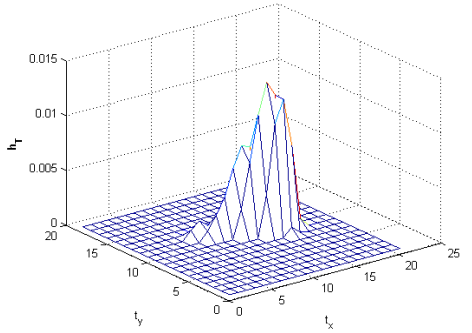

(c)

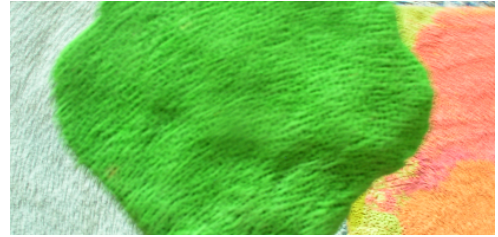

(b)

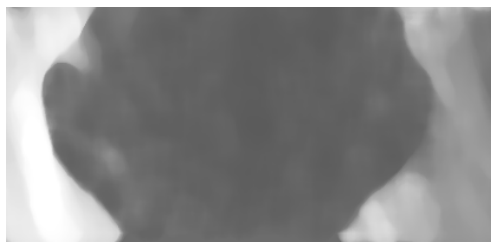

(d)

Figure 5: (a) Reference image. (b) Blurred observation. (c) Estimated TSF at $t_{\theta}=3^{\circ}$ and $t_{s}=1(\mathrm{~d})$ Estimated depth map.

\section{Conclusions}

We initially proposed a technique to estimate the transformations of the reference image that leads to the blurred observation. The method accurately models the blurring effect and can be used for aligning a reference template with its blurred observations. We next developed a technique to extract depth information from motion-blur due to in-plane camera rotations and translations. We used the estimates of the image transformation to obtain the depth information from the translational component of camera motion. Future works can aim to model occlusion affects and allow more degrees-of-freedom of the camera motion.

\section{References}

[1] G. Boracchi. Estimating the 3d direction of a translating camera from a single motionblurred image. Pattern Recognition Letters, 30:671-681, 2009.

[2] G Boracchi, V. Caglioti, and A. Danese. Estimating camera rotation parameters from a blurred image. Proc. The International Conference on Computer Vision Theory and Applications, 2008.

[3] P. Brodatz, 1966. Textures; a photographic album for artists and designers. Dover Publications, New York.

[4] P. Favaro and S. Soatto. A variational approach to scene reconstruction and image segmentation from motion-blur cues. IEEE Conference on Computer Vision and Pattern Recognition, 1:631-637, 2004.

[5] R. Fergus, B. Singh, A. Hertzmann, S. T. Roweis, and W. T. Freeman. Removing 
camera shake from a single photograph. ACM Transactions on Graphics, 25(3):787794, 2006.

[6] J. S. Fox. Range from translational motion blurring. IEEE Conference on Computer Vision and Pattern Recognition, pages 360-365, 1988.

[7] R. Hartley and A. Zisserman. Multiple view geometry in computer vision. Cambridge University Press, 2000.

[8] D. J. Heeger and A. D. Jepson. Subspace methods for recovering rigid motion. International Journal of Computer Vision, 7(2):95-117, 1992.

[9] H. Hong and T. Zhang. Fast restoration approach for rotational motion blurred image based on deconvolution along the blurring paths. Optical Engineering, 42(12):3471, 2003.

[10] S. Julier and J. Uhlmann. A new extension of the Kalman filter to nonlinear systems. The 11th International Symposium on Aerospace/Defense Sensing, Simulation and Controls, pages 182-193, 1997.

[11] G. Klein and T. Drummond. A single-frame visual gyroscope. Proc. British Machine Vision Conference, pages 529-538, 2005.

[12] A. Levin, Y. Weiss, F. Durand, and W. T. Freeman. Understanding and evaluating blind deconvolution algorithms. IEEE Conference on Computer Vision and Pattern Recognition, pages 1964-1971, 2009.

[13] S. Z. Li. Markov random field modeling in computer vision. Springer Verlag, 1995.

[14] D. Lin, E. Grimson, and J. Fisher. Learning visual flows: A lie algebraic approach. IEEE Conference on Computer Vision and Pattern Recognition, pages 747-754, 2009.

[15] H. Y. Lin and C. H. Chang. Depth recovery from motion blurred images. International Conference on Pattern Recognition, 1:135-138, 2006.

[16] C. Mei and I. Reid. Modeling and generating complex motion blur for real-time tracking. IEEE Conference on Computer Vision and Pattern Recognition, pages 1-8, 2008.

[17] C. Paramanand and A. N. Rajagopalan. Unscented transformation for depth from motion-blur in videos. IEEE Workshop on Three Dimensional Information Extraction for Video Analysis and Mining in conjunction with CVPR 2010.

[18] A. A. Sawchuk. Space-variant image restoration by coordinate transformations. J. Opt. Soc. Am., 64(2):138-144, 1974.

[19] Q. Shan, W. Xiong, and J. Jia. Rotational motion deblurring of a rigid object from a single image. IEEE International Conference on Computer Vision, pages 1-8, 2007.

[20] M. Sorel and J. Flusser. Space-variant restoration of images degraded by camera motion blur. IEEE Trans. Imag. Proc., 17(2):105-116, 2008.

[21] Y. Tai, H. Du, M. Brown, and S. Lin. Image/video deblurring using a hybrid camera. IEEE Conference on Computer Vision and Pattern Recognition, pages 1-8, 2008. 
[22] O. Whyte, J. Sivic, A. Zisserman, and J. Ponce. Non-uniform deblurring for shaken images. IEEE Conference on Computer Vision and Pattern Recognition, 2010.

[23] L. Yuan, J. Sun, L. Quan, and H. Y. Shum. Image deblurring with blurred/noisy image pairs. ACM Transactions on Graphics, 26(3):1-10, 2007. 